hep-ph/0405159

SCIPP-2004/15

SU-ITP 04/19

\title{
Visible Effects of the Hidden Sector
}

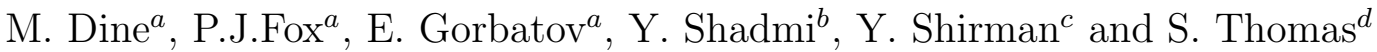 \\ ${ }^{a}$ Santa Cruz Institute for Particle Physics, Santa Cruz CA 95064 \\ ${ }^{b}$ Physics Department, The Technion-Israel Institute of Technology, Haifa 32000, ISRAEL \\ ${ }^{c}$ T-8, MS B285, LANL, Los Alamos, NM 87545 \\ ${ }^{d}$ Physics Department, Stanford University, Stanford, CA 94305
}

\begin{abstract}
The renormalization of operators responsible for soft supersymmetry breaking is usually calculated by starting at some high scale and including only visible sector interactions in the evolution equations, while ignoring hidden sector interactions. Here we explain why this is correct only for the most trivial structures in the hidden sector, and discuss possible implications. This investigation was prompted by the idea of conformal sequestering. In that framework hidden sector renormalizations by nearly conformal dynamics are critical. In the original models of conformal sequestering it was necessary to impose hidden sector flavor symmetries to achieve the sequestered form. We present models which can evade this requirement and lead to no-scale or anomaly mediated boundary conditions; but the necessary structures do not seem generic. More generally, the ratios of scalar masses to gaugino masses, the $\mu$-term, the $B \mu$-term, $A$-terms, and the gravitino mass can be significantly affected.
\end{abstract}




\section{Introduction-A Simple Argument for Conformal Sequester- ing}

Anomaly mediation $[1,2]$ is generally regarded as an interesting, and possibly predictive, scheme for soft supersymmetry breaking parameters. In order that the anomaly-mediated contributions dominate, however, it is necessary that the Kahler potential has a particular, "sequestered" form. Some arguments for how this structure might emerge for geometrical reasons in higher dimensional constructions were given in [2]. However, in many situations in string and $\mathrm{M}$ theory, where one might have hoped to obtain geometric sequestration, the Kahler potential does not have the required form [3]. The dominance of anomaly mediated contributions in geometric realizations seems to require that the underlying microscopic theory possess (nonabelian discrete) symmetries acting on the fields of the visible sector. However, such symmetries, if present, can already provide a solution of the supersymmetric flavor problem.

Luty and Sundrum (LS) [4] have proposed an interesting alternative way of realizing anomaly mediation in four dimensional effective field theories called "conformal sequestering," inspired, in part, by warped constructions in higher dimensions. They argue that in theories in which the hidden sector is conformal over a range of energies, the Kahler potential flows to the sequestered form. In this note, we will review and slightly recast these arguments. We will stress that in the simplest realizations, as noted by LS, additional continuous or discrete symmetries are required to achieve the sequestered form. Amusingly, the symmetries in this case must act on hidden sector fields, as opposed to visible ones. We will then extend the analysis of LS to a class of models with gauge singlet fields which require smaller continuous or discrete symmetries. We also show that conformal sequestering may be achieved without any hidden sector flavor symmetries, but only under rather exceptional circumstances or with specially chosen couplings to hidden sector singlets.

Apart from the question of anomaly mediation, the observations of Luty and Sundrum lead

us to focus on another issue: the role of hidden sector interactions in the renormalization group evolution of operators responsible for visible sector soft-breaking parameters. The standard method to analyze theories with supersymmetry breaking in a hidden sector has been the following. One supposes that the scale of interactions which separates the visible from the hidden sector is $M$. (We have in mind theories where $M$ is large, such as theories of gravity mediation, or gauge mediation with very massive messengers). One writes an effective action for the visible sector fields at the scale $M$ treating the hidden sector fields as non-dynamical background fields. 
This is a supersymmetric theory with higher dimensional operators suppressed by the scale $M$ which couple the visible and hidden sectors. One then writes a set of renormalization group equations for the higher dimensional operators and evolves these into the infrared, ignoring the hidden sector dynamics. Supersymmetry breaking $F$ and $D$ components of the background hidden sector fields then give rise to visible sector soft supersymmetry breaking parameters.

Stated in this way, it is clear that if hidden sector fields are treated as dynamical, then in addition to the usual contributions due to visible sector interactions, hidden sector interactions can also, in general, give rise to appreciable renormalization group evolution of the operators responsible for soft supersymmetry breaking parameters. By considering the evolution of different types of soft masses, one can quickly derive the results of LS, and extend them to a broader class of models. In this note, we will explain this phenomenon first for globally supersymmetric theories, then for locally supersymmetric theories. We will illustrate the point first using a simple theory where it is easy to perform explicit computations, and we will then discuss gauge theories and conformal field theories, both weakly and strongly coupled. We review the analysis of LS, stressing, as they noted, that discrete symmetries are required in their models if anomaly mediation is to dominate. We then go on to construct models with smaller and possibly no symmetry requirements. The general features of hidden sector renormalization on the operators responsible for direct gaugino masses, the $\mu$ and $B \mu$ terms, and $A$-terms are also considered. The classes of models presented here with for conformal sequestering with which do not require microscopic discrete symmetries are shown to yield either anomaly mediated or no-scale boundary conditions. We conclude with an assessment of anomaly mediation, arguing that, while it might occur in specially chosen models of geometric or conformal sequestering, it does not seem to be a generic outcome of any more fundamental framework with which we are currently familiar. But more generally, hidden sector interactions can have an important impact on the spectrum of soft supersymmetry breaking parameters.

\section{Global Supersymmetry}

\subsection{Yukawa theories}

Consider a theory with two sets of fields, $Q$ and $T$, with superpotential

$$
W=\frac{1}{3 !} \lambda T^{3},
$$


where throughout $T$ are understood to be hidden sector fields while $Q$ are visible sector fields. Suppose that at the high scale, $M$, there is also a higher dimension, Kahler potential coupling between the two sectors

$$
\mathcal{L}_{T Q}=c \int d^{4} \theta T^{\dagger} T Q^{\dagger} Q
$$

where $c(M) \sim 1 / M^{2}$.

It is straightforward to compute the anomalous dimension of the operator in Eqn. (2). In terms of supergraphs, there is only one vertex diagram; one also has to include a wave function renormalization of the $T$ fields. The result is

$$
\begin{aligned}
\delta c & =3 c \frac{\lambda^{2}}{16 \pi^{2}} \ln (\mu / M), \\
\frac{d}{d t} c & =3 \frac{\lambda^{2}}{16 \pi^{2}} c .
\end{aligned}
$$

where throughout $t \equiv \log \mu$ is the logarithm of the renormalization scale. One can alternatively derive this result using arguments along the lines of [5]. Think of $Q$ as a background field, and define

$$
Z_{o}=\left(1+c Q^{\dagger} Q\right)
$$

At one loop, $Z$ is corrected; the $T$ propagator is proportional to $Z_{o}^{-1}$. So the self energy correction is proportional to $Z_{o}^{-2}$, and the $T$ effective Lagrangian is [5]:

$$
\mathcal{L}_{T}=Z_{o}\left(1-Z_{o}^{-3} \frac{\lambda^{2}}{16 \pi^{2}} \ln (\mu / M)\right) T^{\dagger} T
$$

which yields the same anomalous dimension for the operator $\mathcal{L}_{t t q q}$.

Let us briefly comment on the background field calculation above. Obviously, the wavefunction coefficient $Z$ is not a measurable quantity, and physics can not depend on the change of normalization for kinetic terms. Thus we need to specify the effect of the redefinition (5) on the canonical coupling $\lambda_{c}=\lambda / Z^{3 / 2}$. To keep the physical coupling unchanged would require a non-holomorphic, field-dependent rescaling of the superpotential coupling $\lambda$. Since, in the limit of interest, $Q$ is a light dynamical field, such rescaling is not consistent with maintaining a manifestly supersymmetric Lagrangian. Therefore, the superpotential coupling $\lambda$ (as opposed to the canonical coupling $\lambda_{c}$ ) must be held fixed.

Now we can consider the implications of this operator for supersymmetry breaking. Following LS we first imagine that the $F$-component of $Q$ has an expectation value. This gives 
rise to a soft mass for $T$,

$$
m_{t}^{2}=c\left|F_{Q}\right|^{2}
$$

According to Eqn. (4),

$$
\frac{d}{d t} m_{t}^{2}=3 \frac{\lambda^{2}}{16 \pi^{2}} m_{t}^{2}
$$

This agrees with the running of the mass predicted in such a theory by the standard analysis; see, e.g. [6].

Now we turn to the case of interest and suppose that that $F_{T} \neq 0$ so that, at $M$,

$$
m_{q}^{2}=c\left|F_{o}\right|^{2}
$$

where $F_{o}=F_{T}(M)$ is the $F$ term vev of $T$ at the messenger scale. Notice that the soft mass is proportional to the vacuum energy in this globally supersymmetric model. As a result of the running of $c$, the $Q$ mass evolves. We need to also think about the renormalization of $F_{T}$. Generally $F_{T}$ will be a function of other fields in the model. If we assume that any non-perturbative renormalization of the superpotential is unimportant, then we can write the Lagrangian as before, but $F_{T}=Z_{T}^{-1 / 2} F_{o}$, where $F_{T}=F_{T}(\mu)$ is the $F$ term for the canonically-normalized field at the scale $\mu$. Furthermore, $F_{o}$ should also be written in terms of the canonically normalized fields at $\mu$. So the $Q$ mass renormalizes with both the running of $c$ and the running of the vacuum energy. Relative to the vacuum energy running of the soft mass is still given by a factor $Z_{T}^{-3}$. If the $\mathrm{T}$ sector has a natural scale (e.g. the scale of supersymmetry breaking in the hidden sector, $\left.M_{h i d}\right)$, then the coefficient only runs down to this scale.

To illustrate this, one can take an O'Raiferataigh type model, with

$$
W=T\left(\lambda_{o} A^{2}-\xi_{o}^{2}\right)+m_{o} B A
$$

where $\lambda_{o}, m_{o}$ and $\xi_{o}$ are "bare" parameters, i.e. parameters of the effective Lagrangian at the scale $M$. For large $m_{o}$, only $T$ develops an $F$-term, $F_{T o}=\xi_{o}^{2}$. At lower scales, this expression can be written in terms of renormalized fields and parameters, where $\xi^{2}=\xi_{o}^{2} / \sqrt{Z_{T}}$. Now suppose that at the scale $M$, the theory contains the operator:

$$
\mathcal{L}_{A Q}=a \frac{1}{M^{2}} A^{\dagger} A Q^{\dagger} Q .
$$

Since there is no $F$ term for $A$, this operator does not give a soft mass for $Q$ at tree level. However, at one loop, it induces the operator of Eqn. (2), with coefficient,

$$
c=2 a \frac{\lambda^{2}}{16 \pi^{2}} \ln (\mu / M) .
$$


(All of the quantities in this expression are renormalized quantities; note that the wave function of $T$ is not important at this order).

So this example is more dramatic: the soft mass vanishes at the scale $M$, but it is induced by the low energy dynamics of the hidden sector under renormalization group evolution to lower scales. Again, note that this operator only runs down to the scale of the $T$ interactions (i.e. roughly to the scale $\xi$ ).

\section{$2.2 \quad$ Gauge theories}

The effects of hidden sector gauge interactions on the running of operators which couple the visible and hidden sectors can also be included. We wish to consider the renormalization of the operator of Eqn. (2). From our analysis of the previous section, we can proceed very simply. If we suppose first, that $F_{Q} \neq 0$, the operator (2) generates a soft mass for the $T$ scalars. In a pure gauge theory, the running of this soft mass is well known:

$$
\frac{d}{d t}\left(m^{2}\right)_{j}^{i}=\frac{1}{16 \pi^{2}}\left(8 \delta_{j}^{i} g^{4} C(i) \operatorname{Tr}\left(S(r) m^{2}\right)\right) .
$$

where $C(i)$ is the Casimir appropriate to the scalar in question; the trace is a sum over all of the matter fields, again with $S(r)$ being the appropriate character of the representation.

So we see that if we write the operator of Eqn. (2) including the most general flavor structure

$$
\mathcal{L}_{t t q q}=\sum_{i j ; a b} c_{i j: a b} \int d^{4} \theta T_{i}^{\dagger} T_{j} Q_{a}^{\dagger} Q_{b}
$$

then, at weak coupling:

$$
\frac{d}{d t} c_{i j: a b}=\frac{1}{16 \pi^{2}}\left(8 \delta_{i j} g^{4} C(i) \operatorname{Tr}\left(S(r) c_{i j: a b}\right)\right)
$$

(the trace is on the $i, j$ indices).

This running can be derived more directly working with Feynman graphs. One particularly simple approach is suggested by a discussion in LS. For simplicity of writing, consider a theory with vanishing superpotential, and $N_{f}$ vectorlike flavors. Study the flavor singlet operator:

$$
\mathcal{L}_{t t q q}=c \sum_{i j} \int d 4 \theta T_{i}^{\dagger} T_{j} Q_{a}^{\dagger} Q_{a}
$$

Various components of this operator may be considered. In particular, one of the terms in this

expression is a product of currents, $j_{T}^{\mu} j_{\mu Q}$. In the limit $c=0$, these currents are conserved. 
Since the $Q$ 's are treated as non-interacting, it is tempting to say that the operator appearing here is not renormalized. But $j_{T}^{\mu}$ is in general an anomalous chiral current. If the theory is regulated with a Pauli-Villars regulator of mass $M_{p v}$, then the renormalization can be determined by noting that because of the conformal anomaly the divergence of the current receives a contribution from the Pauli-Villars fields. The matrix element of the corresponding density $\left(M_{p v} \bar{\psi}_{p v} \psi_{p v}\right)$ is easily evaluated, and gives Eqn. (15).

Note that if we consider an anomaly free hidden sector vector current, there is indeed no renormalization. The same holds for operators which are flavor non-singlet in visible sector fields (as was discussed in [4]).

\section{Local Supersymmetry}

The analysis of theories with local supersymmetry is similar to the analysis of globally supersymmetric theories described above. We first consider the $\lambda T^{3}$ model in the locally supersymmetric case. As an example, we suppose that the Kahler potential is that appropriate to "minimal gravity mediation," i.e it is universal, with only quadratic terms in the chiral fields in the Einstein frame:

$$
K=T^{\dagger} T+Q^{\dagger} Q
$$

(where we have restricted our attention to a model with only these two fields). We will ask, to order $\lambda^{2}$, what sorts of corrections are induced to $K$. Again, we will see that the corrections account for the usual running of the soft masses.

The conventional Einstein frame form for the action is not the most convenient for this analysis. Instead, the standard auxiliary field analysis $[7,8]$ may be done in the superconformal frame, where the Ricci scalar is multiplied by a function $\Phi$, of chiral fields. $\Phi$ is related to the Kahler potential by:

$$
K=-3 \ln (-\Phi / 3)
$$

The Kahler potential of Eqn. (17) corresponds to $\Phi$ which includes

$$
\Phi \supset T^{\dagger} T+Q^{\dagger} Q-\frac{1}{6}\left(T^{\dagger} T+Q^{\dagger} Q\right)^{2} .
$$

In this form, by focusing on particular terms in the component field action, it is easy to compute

the renormalization of the various terms. In particular, the term $T^{\dagger} T Q^{\dagger} Q$ is renormalized by 
the same diagrams (in terms of component fields) as in the globally supersymmetric case. In other words, the function $\Phi$, at one loop in hidden sector fields, has the form:

$$
\begin{aligned}
\Phi & \supset(1+\delta Z) T^{\dagger} T+Q^{\dagger} Q-\frac{1}{3}\left(T^{\dagger} T Q^{\dagger} Q\right)(1-2 \delta Z)+\ldots \\
& \supset T^{\dagger} T+Q^{\dagger} Q-\frac{1}{3}\left(T^{\dagger} T Q^{\dagger} Q\right)(1-3 \delta Z) .
\end{aligned}
$$

where

$$
\delta Z=\frac{\lambda^{2}}{32 \pi^{2}} \ln \left(\mu^{2} / M^{2}\right),
$$

and in the second line we have rescaled (renormalized) the field $T$. The conventional Kahler potential is now given by

$$
K \simeq T^{\dagger} T+Q^{\dagger} Q+(1-3 \delta Z)\left(T^{\dagger} T Q^{\dagger} Q\right)
$$

One can also check that this works properly in the Einstein frame. Some of the simplest terms to study are the quartic fermion couplings; it is easy to isolate them, compute their renormalization, and infer the correction to the Kahler potential. If $F_{Q} \neq 0$, we again reproduce the standard running of the soft mass for $T$. But again, if $F_{T} \neq 0$, we see that the soft masses for $Q$ run in the low energy theory.

\subsection{Are Hidden Sector Dynamics Important?}

In the next section, we will consider the proposal of Luty and Sundrum, that the supersymmetrybreaking sector is a strongly coupled field theory, conformal over a range of energies. In this case, the effects of hidden sector renormalization of the quartic terms in the Kahler potential are profound. First, however, we ask what one might expect the size of these effects to be in asymptotically free hidden sector theories which are weakly coupled at the messenger scale. We will see that even in this case they can easily be of order one or larger.

As an example, we take our "hidden sector" theory to be an $S U(N)$ gauge theory with $N_{f}$ flavors, $T_{i}$ and $\bar{T}_{i}$, and a singlet, $S$. We take the singlet to couple to one of the flavors, with superpotential:

$$
W=\lambda S T_{1} \bar{T}_{1}
$$

The one-loop renormalization group equations for this theory are

$$
\frac{d \lambda^{2}}{d t}=-\frac{c \lambda^{2}}{16 \pi^{2}} g^{2}+2 \frac{N+2}{16 \pi^{2}} \lambda^{4}
$$




$$
\begin{aligned}
\frac{d m_{T}^{2}}{d t} & =\frac{\lambda^{2}}{16 \pi^{2}}\left(2 m_{S}^{2}+4 N m_{T}^{2}\right) \\
\frac{d g^{2}}{d t} & =-\frac{2 b_{o}}{16 \pi^{2}} g^{4}
\end{aligned}
$$

where $m^{2}$ are the soft masses-squared, $c=4\left(N^{2}-1\right) / N$, and $b_{o}=3 N-N_{f}$. The Yukawa coupling has an attractive quasi-fixed point trajectory given by

$$
\frac{\lambda^{2}}{g^{2}}=\frac{c-2 b_{o}}{2(N+2)}
$$

Restricting to this trajectory for simplicity, the one-loop renormalization group equation for $m_{T}^{2}$ are easily solved

$$
m_{T}^{2}(\mu)=m_{T}^{2}(M)\left(\frac{g^{2}(\mu)}{g^{2}(M)}\right)^{\frac{N}{N+2} \frac{2 b_{o}-c}{b_{o}}}
$$

As before, we can interpret this as the renormalization of the coefficient of the operator $T^{\dagger} T Q^{\dagger} Q$, where $Q$ is a visible sector field, which for the purposes of the calculation above may be thought of as having an auxiliary field expectation value. To get a sense of how large the hidden sector renormalization effects can be, consider the exponent in (29). In the large $N$ limit at fixed $N_{f}$ it becomes $\frac{2}{3}$. So if the hidden sector gauge coupling becomes strong, the effects can be sizeable. The expression (29) is only valid at one loop, but the loop expansion only begins to break down for $g^{2}(\mu) N \sim 16 \pi^{2}$, illustrating the possibility of very large effects. Strong hidden sector dynamics is common in renormalizable theories of dynamically broken supersymmetry. So large renormalization effects should be typical of a wide classes of models.

Again, there are a variety of ways one might want to think about these results. Instead of studying the running of operators due to hidden sector interactions starting at the scale $M$, one can alternatively (and equivalently) adopt the viewpoint that one should take boundary conditions for the renormalization group equations, not at scale $M$, but at the lower scale, $M_{\text {hid }}$. Of course, determining these conditions requires knowledge of the microscopic physics at $M$, and the full renormalization group evolution down to $M_{h i d}$.

\subsection{Gravitino Mass}

The gravitino mass is of course directly related to the scale of supersymmetry breaking in the hidden sector. Assuming the cosmological constant vanishes by a cancellation between auxiliary 
and superpotential expectation values, the gravitino mass squared is

$$
m_{3 / 2}^{2}=\frac{3|F|^{2}}{M_{p}^{2}}
$$

where here $|F|^{2}$ is understood to be a sum over all renormalized auxiliary expectation values in the hidden sector. In the notation of section 2.1, the renormalized values of individual expectation values are $F=Z^{-1 / 2} F_{o}$. So effects of hidden sector renormalization appear also in the gravitino mass.

In many models it is important to consider the effects of hidden sector renormalization on visible sector soft parameters relative to the gravitino mass. This is particularly true for models of conformal sequestering since anomaly mediated contributions to soft masses are suppressed by a loop factor compared with the gravitino mass.

\section{The Luty-Sundrum Model}

LS consider the case where the $T$ fields are part of a gauge theory which is nearly conformal over a range of scales. The theories which they consider are required to have a global, nonabelian hidden sector flavor symmetry. They present a sophisticated argument to determine the running of $c$, but the main features of the result are clear from our discussion above. Again suppose first, for illustrative purposes, that $F_{Q} \neq 0$. Then at $M$, the $T$ fields have a non-zero mass. This will exhibit nearly power-law variation with scale since the hidden sector is nearly conformal. Thus the coefficient $c$ of the operator coupling the visible and hidden sectors does as well. In a strongly coupled theory, the anomalous dimension will be of order 1 . Now, as in our perturbative discussion above, we can consider instead $F_{T} \neq 0$, to argue that the soft masses for the $Q$ 's will exhibit power law renormalization since they arise from the same operator.

Actually, the analysis of LS provides further important information: as in our perturbation theory discussion, it demonstrates that hidden sector flavor non-singlet operators have vanishing anomalous dimensions, while a certain hidden sector flavor-singlet operator has anomalous dimension equal to the first derivative of the beta function at the fixed point [4]. Since, by assumption, the fixed point is attractive, this ensures that the anomalous dimension of this operator is positive. If the coefficients of the non-singlet operators vanish, then, in the supergravity context, our analysis of the previous section indicates what happens to $\Phi$ : it behaves as:

$$
\Phi \supset T^{\dagger} T+Q^{\dagger} Q+\mathcal{O}\left(e^{-b^{\prime} t}\right) T^{\dagger} T Q^{\dagger} Q
$$


This is the sequestered form of the Kahler potential. The soft masses arising from direct couplings between the visible and hidden sectors are exponentially suppressed compared with the gravitino mass, and the anomaly-mediated contributions dominate.

In the case of a weakly coupled pure gauge theory (the Banks-Zaks theory [10], to be discussed in more detail below), we can use our earlier arguments to determine the behavior of the various operators. Thinking, again, of the $F_{Q}$ fields as the origin of soft masses for the $T$ fields, we can use the known results for this evolution. At one loop, the anomalous dimensions of the soft masses (in the absence of gaugino masses and Yukawa couplings) vanish; at two loops they are given by Eqn. (13), with $g$ the value of the coupling at the fixed point. Note that one sees immediately that the hidden sector flavor non-singlet operators (14) have vanishing anomalous dimension. The hidden sector flavor singlet operator (14) with $i=j$ flows to zero in the infrared.

As we will see, even at weak coupling, it is easy to construct conformal field theories with much smaller global symmetries, in which almost all of the operator coefficients tend to zero. This is demonstrated by simply introducing soft masses for the hidden sector fields and studying their evolution. Still, in the examples we study below, it is necessary either to impose some discrete symmetry or arrange rather special couplings in the hidden sector in order that anomaly mediation dominate.

In the next subsection, for completeness, we reproduce the LS argument for the anomalous dimension. In the following subsection, we study weakly coupled fixed points, verifying the LS argument, but also developing theories in which all of the soft operators tend to zero.

\subsection{The Anomalous Dimension of the Soft Mass}

We have given a rather explicit derivation of the running of the soft masses in gauge theory. However, there is another argument which generalizes straightforwardly to strongly coupled theories. The basic strategy, following Arkani-Hamed et al [5], is to think of $Z$ as a vector superfield, whose highest component is the soft mass. This is most easily done for the flavor singlet case. We will return to the non-singlet case shortly. One wants to determine the $Z$ dependence of the effective action by studying the renormalization group evolution of the lowest component of $Z$, and then use this to infer the behavior of the highest component. LS consider

a situation where $T T^{\dagger} Q Q^{\dagger}$ should be thought of as giving a correction to $Z_{T}$. They then use this sort of argument to infer soft masses for $Q$. But this can just as well be used to infer soft 
masses for $T$ (due to $F_{Q} F_{Q}^{\dagger}$ ).

In a gauge theory, naively, there is only a uniform rescaling of the kinetic terms, i.e. they are always proportional to $Z_{o}$, the $Z$ factor at the cutoff scale. This is because factors of $Z$ in vertices are always compensated by factors of $Z$ in propagators. Thinking of $Z$ as including the corrections to the Kahler potential, as in Eqn. (5), this would seem to say that the soft masses are not renormalized, since the kinetic terms and soft mass terms would be rescaled by the same factor. On the other hand, we know that at two loops, there is a renormalization of the soft masses.

The resolution of this question was provided by Arkani-Hamed et al. As Shifman et al explained originally [9], the $Z$-dependence of the action is more complicated, because introduction of a regulator introduces new $Z$ dependence. This can be described by relating the physical and holomorphic gauge coupling constants through the relation (valid at all scales)

$$
\frac{8 \pi^{2}}{g^{2}(\mu)}=\frac{8 \pi^{2}}{g_{h}^{2}(\mu)}-N_{c} \ln g^{2}(\mu)-N_{f} \ln Z(\mu)
$$

This relation reconciles the exact NSVZ beta function with the requirement that the coupling constant in the Wilsonian action evolves only at one loop due to the holomorphy. Once wavefunction and holomorphic gauge couplings are promoted to background superfields, the physical coupling must also be treated as a real superfield. Similarly to the case of the Yukawa theories discussed in section (2.1), the holomorphic gauge coupling must be held fixed while the perturbation in the Kahler potential is turned on. This requirement together with Eqn. (32) determines the relation between the perturbation of $\tau=8 \pi^{2} / g^{2}$, and the perturbation of the wave-function, $\delta Z$ :

$$
\delta \tau=\frac{\delta \tau_{h o l}-N_{f} \ln \left(1+\delta_{Z} / Z\right)}{1-N / \tau}
$$

It is easy to see that $\delta \tau$ does not evolve at one loop.

Let's use this to recover the result for the evolution of the soft masses quoted by Martin and Vaughn [6]. The usual solution of the renormalization group equation for $Z(\mu)$ is:

$$
Z(\mu)=Z(M) e^{\int_{g(M)}^{g(\mu)} \frac{d g^{\prime} \gamma\left(g^{\prime}\right)}{\beta\left(g^{\prime}\right)}}
$$

Writing

$$
\beta(g)=-\frac{g^{3} b_{o}}{16 \pi^{2}}, \quad \gamma(g)=c \frac{g^{2}}{16 \pi^{2}}
$$


this gives:

$$
Z(\mu)=Z(M)\left(\frac{g_{p}^{-2}(M)}{g_{p}^{-2}(\mu)}\right)^{-c / 2 b_{o}} .
$$

This is the standard evolution of $Z$ in terms of the gauge coupling of the perturbed theory, $\tau_{p}=\tau+\delta \tau$. Similarly to the Yukawa theory, we can expand the above equation in terms of the perturbation $\delta \tau$. The dependence on the perturbation arises at order $g^{4}-$ as expected it is a two loop effect. Expanding the above in $\delta \tau$ and using the Eqn. (33) gives the correct beta function for the soft mass. This argument is clearly another version of the calculation we described in the previous section.

\subsection{Weakly Coupled Conformal Field Theories}

Let's study the problem of soft masses at a Banks-Zaks fixed point. We will start with a conventional analysis, using the formulas in, say, [6]. The first two beta function coefficients are:

$$
\begin{aligned}
& b_{o}=-\left(3 N-N_{f}\right) \frac{g^{3}}{16 \pi^{2}}, \\
& b_{1}=-\left[6 N^{2}-2 N N_{f}-4 N_{f} \frac{N^{2}-1}{2 N}\right]\left(\frac{g^{2}}{16 \pi^{2}}\right)^{2} g .
\end{aligned}
$$

Requiring that the beta function vanish gives, to this order,

$$
N_{f}=3 N-\epsilon, \quad \frac{g^{* 2}}{16 \pi^{2}}=\frac{\epsilon}{6 N^{2}} .
$$

Here we will think of $N$ as extremely large, and of $\epsilon$ as an integer of order 1 . There will be corrections to this result at higher orders in $g$. In particular, at higher orders, $g^{*}$ is scheme dependent.

Note that near the fixed point,

$$
g=g^{*}+\delta \quad \beta=\beta^{\prime}(g) \delta=b \delta=\frac{\epsilon^{2}}{3 N^{2}} \delta
$$

So

$$
\delta(t)=\delta(0) e^{-b t}
$$

Similarly,

$$
\gamma \approx c=-\frac{g^{2} N}{16 \pi^{2}}=c+d \delta
$$


where $c=-\frac{N}{6} \epsilon ; d=-\frac{2 N}{g} \frac{g^{2}}{16 \pi^{2}}$. The renormalization group equation for $Z$ then leads to:

$$
Z(\mu)=Z(M) e^{c t+\frac{d}{b}(\delta(t)-\delta(0))} .
$$

Indeed, for the Banks-Zaks case the LS argument gives the correct anomalous dimension for the soft masses, $\gamma=\beta^{\prime}$ at the fixed point. The coefficient out front also works out correctly, i.e. one gets the solution with the correct boundary condition to the renormalization group Eqn.

Following LS, we can obtain a general expression for the anomalous dimension, valid at strong coupling. LS ask how the effective action depends on $Z_{o}$. Near the textbook solution of the renormalization group equation to first order in $\delta \tau$, the distance of $\tau$ from the fixed point, is:

$$
Z(t)=\left(Z(0)+\delta_{Z}(0)\right) e^{\gamma^{*} t+\frac{\gamma^{\prime}}{\beta^{\prime}}(\delta \tau(t)-\delta \tau(0))}
$$

where $\gamma^{\prime}$ and $\beta^{\prime}$ are the derivatives of the $\gamma$ and $\beta$ functions with respect to $\tau$. From the NSVZ formula for the $\beta$ function, we also have:

$$
\beta^{\prime}=N_{f} \frac{\gamma^{\prime}}{(1-N / \tau)}
$$

Exploiting the Eqn. (33) we obtain

$$
\delta \tau(t)=\frac{\delta \tau_{h o l}+N_{f} \ln \left(1+\delta_{Z}(0) / Z(0)\right)}{(1-N / \tau)} e^{\beta^{\prime} t} .
$$

Substituting back into the expression for $Z(t)$, the factor of $\delta_{Z}(0) / Z(0)$ cancels, and we are left with:

$$
Z(t)=Z(0) e^{\gamma^{*} t} e^{\ln \left(1+\delta_{Z}(0) / Z(0)\right) \delta \tau(t)}
$$

which reproduces the result for the running of the soft mass of the Banks-Zaks fixed point.

\subsection{Purging Flavor Symmetries}

As discussed by Luty and Sundrum, only a particular operator (14) with $i=j$ which is a flavor singlet under hidden sector flavor symmetry is renormalized in the theory discussed above. The model has an $U\left(N_{f}\right) \times U\left(N_{f}\right)$ hidden sector flavor symmetry at the classical level. All but one of the operators $T_{j} T_{j}^{\dagger}$ contain, as their $\theta \bar{\theta}$ components, non-anomalous conserved currents, and 
are thus not renormalized. The one exception is the anomalous axial $U(1)$. As discussed above, it is easy to derive the renormalization of this operator at two loops from the anomaly. If one is to obtain anomaly mediation, it is then necessary to suppress all the operators (14) which are flavor non-singlet in the hidden sector at the high scale. In [4], this is achieved through intricate hidden sector discrete symmetries.

But our earlier discussion suggests that if we can construct a hidden sector CFT without flavor symmetries, it should be relatively easy to suppress all operators (14) connecting the hidden and visible sectors. As discussed below, it is rather difficult to construct conformal models with only gauge-non-singlet fields and without continuous non- $R$ global symmetries. But this can be accomplished if hidden sector gauge singlet fields are included with appropriate superpotential couplings. As an example, consider supersymmetric $S U(N)$ QCD with $N_{f}$ flavors and $N$ colors in the conformal window $\frac{3}{2} N \leq N_{f}<3 N$. We add $N_{f}$ singlets, $S_{i}$, with tree level superpotential

$$
W=\lambda \sum_{i=1}^{N_{f}} S_{i} T_{i} \bar{T}_{i}
$$

which also results in an interacting conformal theory. To achieve such a coupling, one can impose an $S_{N_{f}}$ permutation symmetry, or - even better - simply note that the equations for the fixed point have such a symmetry at one loop. Before writing down explicit formulas note that $\lambda$ has an attractive fixed point, this can be easily verified at weak coupling. As a result, ignoring contributions from hidden sector gaugino masses, many soft masses run to zero (this is a corollary of a theorem of Nelson and Strassler [11]). However, the model has $N_{f}$ vectorlike conserved $U(1)$ 's corresponding to the Cartan sub-algebra of the vector $S U\left(N_{f}\right)$ and the overall vector $U(1)$. Correspondingly, these $N_{f}$ linear combinations of masses (or more precisely, coefficients of quartic operators) do not run.

This models respects an $S_{N_{f}}$ permutation symmetry at the fixed point, as well as a $Z_{2}$ charge conjugation symmetry which exchanges $T_{i} \leftrightarrow \bar{T}_{i}$. If we suppose that this discrete $S_{N_{f}} \times Z_{2}$ symmetry is exact in the microscopic theory, then the dangerous operators would be absent, and anomaly mediation would dominate. The requisite number of symmetries may be reduced further by introducing additional singlets with different couplings to the quarks. For example, adding $N_{f}-1$ additional singlets, $S_{i}^{\prime}$, with tree level superpotential

$$
W=\lambda^{\prime} \sum_{i=1}^{N_{f}-1} S_{i}^{\prime} T_{i} \bar{T}_{i+1}
$$

leaves the overall vector $U(1)$ as the only non-anomalous non- $R$ continuous symmetry. However, 
for $S U(N)$ gauge group with hidden sector flavors, the most general singlet superpotential couplings to mesons which preserve conformal invariance can not eliminate the overall vector $U(1)$ symmetry. So in this class of models at least an exact discrete $Z_{2}$ symmetry in the hidden sector is required in order to suppress all the dangerous operators (14) and obtain conformal sequestering.

The requirement of an exact $Z_{2}$ symmetry can be relaxed in special circumstances. For $S U(N)$ gauge group with flavors this possibility arises only for the special cases of $N=4$, $N_{f}=8$ and $N=6, N_{f}=9$. In these cases baryon and anti-baryon operators with any flavor structure are exactly marginal and may be added to the superpotential without spoiling conformal invariance. For example, for the $S U(4)$ with $N_{f}=8$ theory the superpotential

$$
W=\lambda^{\prime \prime}\left(T_{1} T_{2} T_{3} T_{4}+\bar{T}_{1} \bar{T}_{2} \bar{T}_{3} \bar{T}_{4}\right)
$$

breaks the overall vector $U(1)$. With inclusion of a sufficient number of singlets with couplings to mesons such as (48) and (49), all continuous non- $R$ symmetries are then broken. For $S O(N)$ and $S P(N)$ gauge groups with flavors, all the flavor symmetries are axial. So for these two classes of theories with sufficiently many singlets, superpotential couplings of the form (48) and (49) are alone sufficient to eliminate all continuous non- $R$ symmetries without requiring any microscopic discrete symmetries.

In the absence of gauge singlet fields it is rather difficult to construct a single gauge group CFT which does not possess non- $R$ continuous symmetries at the infrared conformal fixed point. A number of requirements must be met to obtain such a theory. First, in the theory with vanishing superpotential there must exist at least one chiral operator which transforms under each non-anomalous continuous non- $R$ symmetry. Second, at least one operator for each symmetry must be exactly marginal with $U(1)_{R}$ charge $R=2$. This is a rather non-trivial requirement since it is often the case that operators which break global symmetries are in fact (marginally) irrelevant at a fixed point so that there are enhanced global symmetries in the infrared. And third, each such operator which breaks each symmetry must appear in the superpotential of the interacting theory after including the most general set of field redefinitions. Taken together these requirements are rather non-trivial and we expect that they are all satisfied only in rather exceptional circumstances. However, one CFT which does appear to satisfy all these requirements is supersymmetric $S U(4)$ QCD with $N_{f}=8$ flavors. As mentioned above the baryon and anti-baryon chiral operators of this theory are exactly marginal for any flavor structure, so adding these to the superpotential can break flavor symmetries without spoiling 
conformal invariance. For example, the superpotential couplings

$$
W=\lambda \sum_{i=1}^{8}\left(T_{i} T_{i+1} T_{i+2} T_{i+3}+\bar{T}_{i} \bar{T}_{i+1} \bar{T}_{i+2} \bar{T}_{i+3}\right)
$$

break the $S U(8) \times S U(8) \times U(1)$ non-anomalous symmetry to $U(1)^{12}$. And the superpotential couplings

$$
\begin{aligned}
W=\lambda^{\prime} \sum_{i=1}^{4}( & T_{i} T_{i+2} T_{i+3} T_{i+4}+T_{i} T_{i+1} T_{i+3} T_{i+4}+T_{i} T_{i+1} T_{i+2} T_{i+4} \\
& \left.+\bar{T}_{i} \bar{T}_{i+2} \bar{T}_{i+3} \bar{T}_{i+4}+\bar{T}_{i} \bar{T}_{i+1} \bar{T}_{i+3} \bar{T}_{i+4}+\bar{T}_{i} \bar{T}_{i+1} \bar{T}_{i+2} \bar{T}_{i+4}\right)
\end{aligned}
$$

break these remaining symmetries. So in this theory with at least these superpotential couplings, all the operators (14) are suppressed by hidden sector conformal dynamics and conformal sequestering results without any microscopic discrete symmetries.

We leave to future work the construction of all CFT's without continuous non- $R$ symmetries; but conclude that without rather exceptional circumstances and/or without specially chosen couplings possibly to gauge singlet fields, such CFT's do not appear to be generic.

\section{Gaugino Masses and Other Soft Parameters}

Hidden sector renormalization can in principle affect any of the operators responsible for visible sector soft supersymmetry breaking parameters, including not only scalar masses but gaugino masses and the $\mu$ - and $B \mu$-terms. Gaugino Majorana masses require that a gauge singlet field acquire an auxiliary expectation value. This can be the conformal compensator of the supergravity multiplet (as is assumed to dominate in anomaly mediation) or gauge singlet fields directly in the hidden sector. Below we consider the effects of hidden sector renormalization on gaugino mass contributions from the latter type of singlets. This is particularly relevant to the class of nearly conformal models introduced in the previous subsection in which hidden sector gauge singlets are introduced in order to relax the requirement of hidden sector flavor symmetries.

The effective operators which yield gaugino masses from direct couplings to the hidden sector are of the form

$$
c_{\lambda} \int d^{2} \theta S W^{\alpha} W_{\alpha}
$$


where $S$ is a hidden sector singlet, $W_{\alpha}$ is a visible sector superfield strength, and $c_{\lambda}(M) \sim 1 / M$. An auxiliary expectation value $F_{S} \neq 0$ gives a gaugino mass at renormalization scale $\mu$ of

$$
m_{\lambda}(\mu)=c_{\lambda}(\mu) F_{S}(\mu)
$$

Since the operator (53) is linear in the singlet, $S$, the gaugino mass (54) is renormalized by hidden sector dynamics only through the wave function of $F_{S}$. From the discussion of section 2.1 , the renormalized auxiliary expectation value is $F_{S}(\mu)=Z_{S}^{-1 / 2} F_{S, o}$ where $F_{S, o}=F_{S}(M)$ and $Z_{S}(M) \equiv 1$. So the gaugino mass is

$$
m_{\lambda}(\mu)=c_{\lambda}(\mu) \frac{F_{S, o}}{Z_{S}^{1 / 2}}
$$

Written in this way it is clear that the coefficient $c_{\lambda}$ is renormalized only by visible sector dynamics. As an aside, note that unitarity requires the renormalized wave function factor of any gauge singlet field to be greater than one, $Z_{S} \geq 1$. The renormalized auxiliary expectation value appearing in the gaugino mass (55) also appears in the gravitino mass (30) which is likewise renormalized by hidden sector dynamics only through wave function effects. So we see that if supersymmetry breaking is dominated by gauge singlet auxiliary expectation values then hidden sector interactions do not modify the operators responsible for direct gaugino soft masses relative to the gravitino mass. This is in contrast to the operators (2) responsible for scalar soft masses which can be either be suppressed or enhanced by hidden sector renormalization effects, as illustrated in sections 2.1 and 3.1 respectively. This is also in contrast to visible sector renormalization of the operator (53) which is either positive or negative depending on whether the gauge group is asymptotically free or not.

It is also possible to consider the effects of hidden sector renormalization on the operators responsible for the Higgs sector $\mu$ - and $B \mu$-terms. The superpotential $\mu$-term, $W=\mu H_{u} H_{d}$, can arise from hidden sector supersymmetry breaking with an operator of the form

$$
c^{\prime} \int d^{4} \theta S^{\dagger} H_{u} H_{d}
$$

where $c^{\prime}(M) \sim 1 / M$. Like the gaugino mass operator (53) this operator is only linear in the hidden sector singlet $S$. So from the discussion above it follows that if supersymmetry breaking is dominated by gauge singlet auxiliary expectation values then hidden sector interactions do not modify the operator responsible for the $\mu$-term relative to the gravitino mass. The soft supersymmetry breaking Lagrangian $B \mu$ term, $\mathcal{L}=B \mu H_{u} H_{d}$, can arise from an operator of the form

$$
c^{\prime \prime} \int d^{4} \theta T^{\dagger} T H_{u} H_{d}
$$


where $c^{\prime \prime}(M) \sim 1 / M^{2}$. This operator is bi-linear in fields which either transform under hidden sector gauge interactions or are gauge singlet, and is of the general form of the operators (2) responsible for soft scalar masses. So hidden sector interactions can in principle either suppress or enhance the operators responsible for the direct $B \mu$-term relative to the gravitino mass.

Finally, it is also possible to consider the effects of hidden sector renormalization on the operators responsible for scalar tri-linear $A$-terms. These terms arise from operators of the form

$$
c_{A} \int d^{4} \theta S \Phi \Phi \Phi
$$

where here $\Phi$ are understood to be visible sector chiral multiplets and $c_{A}(M) \sim 1 / M$. The specific prefactors of the operators (58) may depend on the underlying theory of flavor. Like the gaugino mass (53) and $\mu$-term (56) these operators are also linear in the hidden sector singlet $S$. So from the discussions above if follows that if supersymmetry breaking is dominated by gauge singlet auxiliary expectation values then hidden sector interactions do not modify the operators responsible for A-terms relative to the gravitino mass.

There are clearly various possibilities for how hidden sector interactions might renormalize the different operators which couple to the visible sector. So small, moderate, or even large ratios of different types of soft supersymmetry breaking parameters could result, depending on details of the hidden sector.

One possibility for large ratios of direct soft parameters to the gravitino mass is the LutySundrum class of models [4]. The hidden sector is assumed to contain only gauge non-singlet fields and flow to an attractive conformal fixed point. As discussed in section 4.3, with appropriately chosen discrete symmetries or a conformal sector without non- $R$-continuous symmetries, the direct scalar masses are vanishingly small. From the discussion above we see that the direct $B \mu$ - term is also vanishingly small. And since there are no hidden sector singlets, the direct gaugino masses, $\mu$-term, and $A$-terms also vanish at leading order. Anomaly-mediated contributions to both scalar and gaugino masses then dominate. The $\mu$ - and $B \mu$-terms must be generated by additional interactions within the visible sector. This class of models, however, suffers the problem of a tachyonic slepton endemic to all models of anomaly-mediated supersymmetry breaking. This unacceptable feature might be cured by yet additional visible sector interactions.

An interesting ratio of direct soft parameters arises for the models of the section 4.3 with a hidden sector with an attractive conformal fixed point, and with sufficiently many singlets with special couplings which break all the continuous non- $R$-symmetries. In this case, if hidden 
sector singlets dominate the supersymmetry breaking auxiliary expectation values, then from the discussion above, for general operators coupling the visible and hidden sectors the gaugino masses, $\mu$-term, and $A$-terms are of order the gravitino mass, $m_{\lambda} \sim \mu \sim A \sim m_{3 / 2}$, while the direct scalar masses and $B \mu$-term are vanishing small, $m_{\phi}^{2}, B \mu \ll m_{3 / 2}^{2}$. The leading direct contributions to the scalar masses are anomaly-mediated and therefore loop-suppressed compared with the gauginos masses. So this class of models yields an interesting set of no-scale boundary conditions. Scalar masses are generated mainly from renormalization group effects

in the visible sector proportional to gaugino masses and possibly $A$-terms. Aside from the $H_{u}$ mass, all scalar masses are then non-tachyonic. And the $B \mu$-term is likewise generated by visible sector renormalization effects proportional to the product of gaugino masses and $\mu$-term. Note that these models yield a phenomenologically viable set of boundary conditions for the correct pattern of electroweak symmetry breaking without postulating additional visible sector interactions to modify soft parameters. However, since the $A$-terms transform under visible sector flavor symmetries, this class of models does not necessarily solve the supersymmetric flavor problem without an underlying theory of flavor.

\section{Conclusions}

From the discussions of the previous sections, we draw two lessons. The first lesson has to do with the idea of conformal sequestering. In general, in a conformal field theory, we might expect large corrections to the operators which generate soft masses. LS have provided an elegant analysis which relates the anomalous dimensions of these operators to the derivative of the beta function at the fixed point, in a certain class of theories. But the present analysis also indicates that generic conformal theories possess continuous non- $R$ global symmetries and therefore do not lead, in any general way, to sequestering. Many of the dangerous operators which could violate visible sector flavor symmetries for example, simply do not run. One must suppose that these operators are absent from the microscopic theory. But in a theory like string theory which contains gravity, there are no continuous global symmetries, so one must suppose that the absence of these terms results from hidden or visible sector discrete symmetries, with a very special structure. Indeed, in the original Luty-Sundrum model, an intricate set of hidden sector discrete symmetries are imposed in order to obtain sequestering. However, we have seen that it is fact possible to obtain conformal theories without non- $R$ continuous symmetries, but only in rather exceptional cases or with specially chosen couplings to gauge singlet fields. In the end, it is not clear to us that this can be viewed, in any sense, as a generic outcome to be 
expected of some sort of more fundamental theory. Only a full survey of such theories might give an indication.

We view this result as a positive one. In general, in thinking about Beyond the Standard Model physics, there are few limits to our speculations. But the realization that geometrical separation does not lead, generically, to sequestering suggests that anomaly mediation is not a likely outcome of any fundamental theory, such as known string models. Conformal sequestering, as originally proposed, requires the same sorts of discrete symmetries as geometric sequestering, if it is to dominate. We have seen that it may be possible to achieve such sequestering without discrete symmetries, but this phenomenon does not seem generic.

The second lesson is quite general: the evolution of the operators responsible for soft parameters cannot be determined without knowledge of hidden sector dynamics. It is not enough to start with knowledge of the operators which couple the hidden and visible sectors at, say, the Planck scale and evolve these down to low energies using only visible sector dynamics. Hidden sector dynamics will also lead to evolution of the operators. If the hidden sector is, as one might imagine, strongly coupled in order to break supersymmetry, these effects could be quite large. The Luty-Sundrum model is an extreme example of this phenomenon. Alternatively, and equivalently, it is necessary to provide boundary conditions for the renormalization group equations for the operators, not at the scale $M$, but at the scale at which the hidden sector decouples, $M_{\text {hid }}$.

It should be remarked that the importance of hidden sector dynamics discussed in this paper is an issue for the Wilsonian approach to renormalization, where by definition dynamics in both the hidden and visible sectors are integrated out scale by scale. In this approach the hidden sector can affect physics over all scales down to $M_{h i d}$ and must be taken into account in the running of the non-renormalizable operators which couple the hidden and visible sectors. However, since the hidden and visible sectors are by definition not coupled through renormalizable interactions, the effects of each sector on the running of non-renormalizable operators factorize. It is then possible, in principle, to integrate out the hidden sector completely and arrive at a 1PI effective action for visible sector fields alone. In this case, the presence of nontrivial hidden sector dynamics can be subsumed into the boundary conditions for the visible sector soft parameters at the messenger scale $M$. In this approach, hidden sector dynamics does not directly affect the renormalization group running of visible sector parameters below the messenger scale $M$. But, as discussed here and as is apparent in the Luty-Sundrum model, it can have an important impact on the relative magnitude of different soft parameters at the 
high scale. In particular ratios of scalar masses to gaugino masses, the $\mu$ term, the $B \mu$ term, $A$-terms, and the gravitino mass, can be significantly affected.

\section{Acknowledgements:}

We thank T. Banks, T. Bhattacharya, A. Friedland, M. Luty, E. Poppitz, R. Sundrum and J. Terning for conversations. The work of M.D., P.F. and E.G. was supported in part by the U.S. Department of Energy. M. Dine also acknowledges support from the U.S.-Israel Binational Science Foundation. Y. Shadmi thanks UCSC-SCIPP and the SLAC theory group for hospitality while this work was initiated. The research of Y. Shadmi is supported in part by the Israel Science Foundation (ISF) under grant 29/03. The research of Y. Shadmi and Y. Shirman was supported in part by the United States-Israel Binational Science Foundation (BSF) under grant 2002020. Y. Shirman thanks UCSC-SCIPP and Physics Department at Technion for hospitality during the work on this project. Y. Shirman is a Richard P. Feynman fellow at Los Alamos National Laboratory and is supported by the US Department of Energy. The work of S.T. was supported in part by the US National Science Foundation under grant PHY02-44728.

\section{References}

[1] G. F. Giudice, M. A. Luty, H. Murayama and R. Rattazzi, JHEP 9812, 027 (1998) [arXiv:hep-ph/9810442].

[2] L. Randall and R. Sundrum, Nucl. Phys. B 557, 79 (1999) [arXiv:hep-th/9810155].

[3] A. Anisimov, M. Dine, M. Graesser and S. Thomas, JHEP 0203, 036 (2002) [arXiv:hepth/0201256]. Phys. Rev. D 65, 105011 (2002) [arXiv:hep-th/0111235].

[4] M. Luty and R. Sundrum, Phys. Rev. D 67, 045007 (2003) [arXiv:hep-th/0111231]. Phys. Rev. D 65, 066004 (2002) [arXiv:hep-th/0105137].

[5] N. Arkani-Hamed, G. F. Giudice, M. A. Luty and R. Rattazzi, Phys. Rev. D 58, 115005 (1998) [arXiv:hep-ph/9803290].

[6] S. P. Martin and M. T. Vaughn, Phys. Rev. D 50, 2282 (1994) [arXiv:hep-ph/9311340].

[7] E. Cremmer, S. Ferrara, L. Girardello and A. Van Proeyen, Nucl. Phys. B 212, 413 (1983).

[8] J. Wess and J. Bagger, Supersymmetry and Supergravity, Princeton University Press (Princeton) 1992. 
[9] V. A. Novikov, M. A. Shifman, A. I. Vainshtein and V. I. Zakharov, Nucl. Phys. B 229, 381 (1983).

[10] T. Banks and A. Zaks, Nucl. Phys. B 196, 189 (1982).

[11] A. E. Nelson and M. J. Strassler, JHEP 0207, 021 (2002) [arXiv:hep-ph/0104051]. 